\title{
Los viajes como rupturas transformadoras de las memorias sociales territorializadas
}

\section{Travels as transformative ruptures of territorialized social memories}

\section{Nadia Tamara Chiaravalloti \\ nadiachiaravalloti@gmail.com \\ Universidad Nacional de Luján, Argentina \\ Universidad de Buenos Aires, Argentina}

Recepción: 29 Junio 2021

Aprobación: 15 Octubre 2021

Publicación: 01 Noviembre 2021

Cita sugerida: Chiaravalloti, N. T. (2021). Los viajes como rupturas transformadoras de las memorias sociales territorializadas. Anuario del Instituto de Historia Argentina, 21(2), e148. https://doi.org/10.24215/2314257Xe148
Resumen: El presente trabajo se enmarca en la investigación en curso para la finalización de la tesis de licenciatura en Ciencias Antropológicas, orientación sociocultural. El objetivo es historizar la Educación Intercultural Bilingüe (EIB) en la Argentina, a partir de la biografía de vida de Marta Tomé, educadora, pionera y referente de la temática. A la luz de uno de los puntos relevantes con el que nos encontramos en la investigación, retomamos para este artículo los viajes que la docente realizó, a lo largo de su juventud, por varios países de Latinoamérica. Estos representan rupturas transformativas que influyeron en su militancia y su compromiso por la educación. En la actualidad, forman parte de su identidad y su memoria, que no pueden disociarse de la memoria social, colectiva y co-construida. El trabajo metodológico realizado a partir del enfoque de la biografía de vida permitió dar cuenta de que estos viajes forman parte de las prácticas interculturales que se generaron en diversos territorios, y que hoy hacen a la memoria social y territorializada de la educación intercultural en la Argentina.

Palabras clave: Viajes, Memorias, Territorios, Identidades, Biografía de vida.

Abstract: The present paper is part of the ongoing research for the completion of the Bachelor thesis in Anthropological Sciences, sociocultural orientation. The objective is to historicize Intercultural Bilingual Education (EIB) in Argentina, based on the life biography of Marta Tomé, educator, pioneer and benchmark on the subject. In the light of one of the relevant points that we find in the research, we get back for this article, the journeys that the teacher made throughout her youth, through various Latin American countries. These represent transformative ruptures that influenced their militancy and commitment to education. At present, they are part of their identity and memory, which cannot be dissociated from the social, collective and co-constructed memory. The based methodological work carried out from the approach of the life biography allowed to realize that these journeys are part of the intercultural practices that were generated in various territories, and that today make up the social territorialized memory of intercultural education in Argentina.

Keywords: Travels, Memories, Territories, Identities, Life biography. 


\title{
INTRODUCCIÓN
}

Marta Tomé es una educadora argentina que ha trabajado con diversas comunidades educativas en distintos territorios, como el Conurbano y las provincias de Santa Fe, Chaco, Corrientes, Neuquén y Río Negro. En la investigación que llevamos a cabo, junto a la docente buscamos recuperar las líneas de trabajo sobre educación intercultural durante la década de los años setenta. En este caso, el foco que tratamos de analizar se encuentra en los distintos viajes que realizó por Latinoamérica, que fueron rupturas transformativas en su vida, su compromiso por la educación y su militancia en el Tercermundismo.

Asimismo, en la década mencionada viaja a la provincia de Chaco, donde desarrolla la metodología de bialfabetización y pareja pedagógica. El trabajo cooperativo que organizaba junto con las comunidades de El Impenetrable, más específicamente en la zona de El Sauzalito, lleva a la creación de una escuela-rancho y una currícula que se decidía en función de las distintas parejas pedagógicas y de los diferentes grupos etarios. Las temáticas que se trabajaban tenían relación con las problemáticas del lugar, que giraban en torno a los aportes en alfabetización que en ese momento estaba realizando Paulo Freire (1921-1997). La pareja pedagógica permitió el trabajo con actividades que tuvieran en cuenta las lenguas wichí-español. Lo que se generaba en la escuela-rancho con los distintos grupos es lo que aquí denominamos prácticas educativas interculturales, que consideramos forman parte de los antecedentes de la historización de la educación intercultural en la Argentina.

El desafío que presenta esta investigación es dar cuenta de cómo la educadora Marta Tomé parte de ciertos hitos de su vida para llegar a plantear la metodología de bialfabetización y pareja pedagógica. Los viajes por Latinoamérica que esta docente realizó y la cultura material que se encuentra entramada en su narrativa hacen a la construcción de la historización de la educación intercultural. Los desplazamientos que se dieron desde distintas prácticas en territorio forman parte de las memorias territorializadas (Gómez, 2000). Recuperamos a través de la biografía de vida y de la cultura material cómo comienza a entenderse, desde múltiples matrices, la construcción de otras formas de conocer.

\section{LA BIOGRAFÍA DE VIDA ENTRAMADA EN LAS MEMORIAS SOCIALES TERRITORIALIZADA}

\author{
“(...) pensé en ir a ver a mi tía. Con la excusa de visitar \\ a mi tía ... en realidad era hacer un viaje. Y me mandé un \\ viaje al estilo Che Guevara. Y conoci Bolivia y Perú. Al \\ año siguiente la continué con México y en una de las escalinatas \\ que estábamos haciendo en las ruinas de los \\ aztecas, me dije: acá se terminó el turismo y empieza el \\ compromiso" (Registro de campo: I Jornadas: \\ Interculturalidad y educación: Propuestas desde la \\ diversidad cultural. Universidad Nacional de General \\ Sarmiento, lunes 22 de octubre de 2018).
}

Las prácticas espaciales de los viajes y las prácticas temporales de la escritura etnográfica a través de las biografías de vida son relevantes para la definición y documentación de diversas problemáticas sociales (Clifford, 1999). En la relación entre sujetos/as y los distintos desplazamientos que realizan por los espaciostiempos comienzan a conformarse las memorias sociales territorializadas. En este sentido, la etnografía multilocal (Marcus y Fischer, 1986) es relevante. El trabajo de campo multilocal es una conjunción de espacialidades y temporalidades que desde las biografías de vida implican no lugares, lugares de recuerdos y paisajes que se traen al presente. Es decir que, si bien el trabajo de campo, en este caso, se realiza con la educadora Marta Tomé, en lo que consideramos como parte de las espacialidades a la hora de narrar determinados hitos de su vida, nos desplazamos a: 1) distintos no lugares que implican recorrer las 
temporalidades que atraviesa su trayectoria de vida mediante los acontecimientos transcurridos;2) lugares de recuerdos, es decir lugares que visitamos, pero que se modificaron en relación a un momento que la educadora vivió con anterioridad; 3 ) los paisajes y objetos que hacen a las rupturas transformadoras, y por ende a las distintas formas de conocer y a la construcción de conocimientos.

La biografía de vida (Bourdieu, 1989; Piña, 1989; Bertaux, 2005; Myerhoff, 2007) se encuentra atravesada por objetos, lugares y paisajes que forman parte de las rupturas transformadoras que se entraman en la memoria social, colectiva y territorializada de la Educación Intercultural Bilingüe (EIB) en la Argentina. El trabajo de campo, definido por las prácticas espaciales de viajes, las interpretaciones, observaciones, interacciones y las acciones corporizadas, hacen que se establezca un compromiso social y educativo.

En torno a lo expresado, Tim Ingold sostiene que la idea de paisaje es el mundo conocido, tal como es para aquellos que lo habitan, que viven en sus lugares y viajan a través de sus caminos conectándolos (Ingold, 2013). El paisaje se constituye como un itinerario de vida que tiene relación con las actividades, compromisos, formación, trabajos, familias, militancias, que habitaron espacios y tiempos y que al hacerlo dejaron algo de sí mismos. El paisaje es cualitativo y heterogéneo. Esto queda representado en las formas, los sentires y pensamientos que aparecen a la hora de documentar memorias sociales territorializadas.

El adoptar una perspectiva de este tipo implica situar el conocimiento surgido de la experiencia inmediata, haciendo foco en la escucha de los hitos que las personas narran de su implicación vivida y diaria en el mundo. A partir de este contexto relacional de la vinculación de las personas con el mundo, en el hecho de habitar, cada lugar representa su impronta. En este sentido, entendemos que mientras en los espacios los significados se encuentran unidos al mundo, en el caso del paisaje estos significados son recuperados del mundo (Ingold, 2013). De acuerdo con Ingold, el paisaje parece ser lo que vemos a nuestro alrededor. Sin embargo, el autor define paisaje de tareas (task scape) como lo que escuchamos. Ver el paisaje implica observarlo desde nuestra matriz óptica y cultural, lo cual genera distintas formas de conocer. Ahora bien, el paisaje de tareas presupone la presencia de un agente que mira y escucha. El paisaje de tareas debe estar poblado por seres que sean agentes en sí mismos, y que actúen recíprocamente en el proceso de su propio habitar; es decir, da cuenta de distintas ontologías que se encuentran en nuestro entorno y no está disociado de los entornos de otros agentes (Ingold, 2013). En este caso, el paisaje no deja de ser un agente que se encuentra construido por historias que se construyen en el habitar. Es histórico y dinámico. No se encuentra escindido del tiempo, sino que se va resignificando a lo largo del tiempo. Esto permite los diversos usos y apropiaciones que podemos darle desde el presente histórico situado.

En torno a lo dicho, cabe destacar que los viajes se encuentran conformados por paisajes donde se presentan determinados objetos que tienen su propia biografía, es decir su vida social (Appadurai, 1996), que abarca las vidas y los tiempos de los antecesores que, a lo largo de las generaciones, se movieron alrededor de él y participaron de su formación. El sentipensar (Escobar, 2014) del paisaje y de los objetos que hacen a la cultura material es llevar adelante un acto de rememoración; recordar no es tanto una cuestión de buscar una imagen interna, almacenada en la mente, sino más bien vincularse perceptualmente desde otros lugares con un ambiente que está impregnado de ese pasado-presente.

\title{
LOS VIAJES COMO RUPTURAS TRANSFORMATIVAS
}

\author{
“(...) ese conocimiento a mi me abrió América” (Registro \\ de campo: I Jornadas: Interculturalidad y educación: \\ Propuestas desde la diversidad cultural. Universidad \\ Nacional de General Sarmiento, lunes 22 de octubre de 2018).
}

Los viajes que Marta Tomé realiza a fines de la década de 1960 por Bolivia y Perú (1967) y México (1968) le permitieron conocer otras realidades sociales, desde las cuales se cuestiona muchas de las acciones políticas de su realidad cotidiana, aquella que se construye a partir de los lugares que habita en relación con sus primeros años de vida, que se entraman con su entorno familiar, militante y de formación. Sin embargo, a la hora de 
conocer otros lugares, y en este caso, a partir de los viajes que realiza, conoce otros paisajes y objetos que los conforman y hacen a distintas rupturas que llevan a otras formas de conocer.

M: (...) ese verano me fui a conocer a una tía mía que vivía en Perú, que era monja. Entonces recorrí Bolivia y Perú. Y ahí me enteré de lo que era América Latina. O por lo menos empecé a enterarme (Registro de campo, 30 de noviembre de 2019. Espacio: Taller de Quichua Caypi Quichuapi 'Rimaycu, Casa de la Cultura, partido de Moreno, Buenos Aires).

En el fragmento citado, Tomé relata cómo comienza a ver las problemáticas de América Latina mediante una charla que se realizó en la Casa de la Cultura en el partido bonaerense de Moreno. El hecho de que Marta Tomé relate sus experiencias de vida en este espacio es relevante para aquellas personas que la escuchan, y que trabajan en la memoria y la identidad presente sobre la lengua y cultura quichua. En este sentido, es interesante señalar que hay una configuración de una temporalidad y un espacio distintos de los habituales, en los que se sistematizó la mayor parte de sus experiencias de vida. En otra de las entrevistas, que realizamos en Villa Lugano, Tomé comenta:

M: Y así viajé por Bolivia y Perú, y entonces conocí otra realidad que no tenía nada que ver con la mía. Porque yo acá en la Argentina tuve ocasión también de ir al Valle de Río Negro, pero donde había montón de italianos, entre ellos el primo de mi papá, y también en la provincia de Buenos Aires, en la que también había una familia italiana. O sea, esas dos posibilidades habían tenido (Entrevista, sábado 9 de noviembre de 2019. Espacio: Casa de Marta Tomé, Villa Lugano).

De acuerdo con Krotz (1991), los viajes no implican sólo movimiento en el espacio sino también en el tiempo. La categoría de viaje en antropología se encuentra ligada a aquellas formas que los viajeros utilizaron para conocer otras culturas. En este caso, el viaje es visto a partir de la categoría drama social propuesta por Víctor Turner (2002). Los dramas sociales son unidades de procesos armónicos o inarmónicos que surgen en situación de conflicto, aunque también pueden darse en otras situaciones.

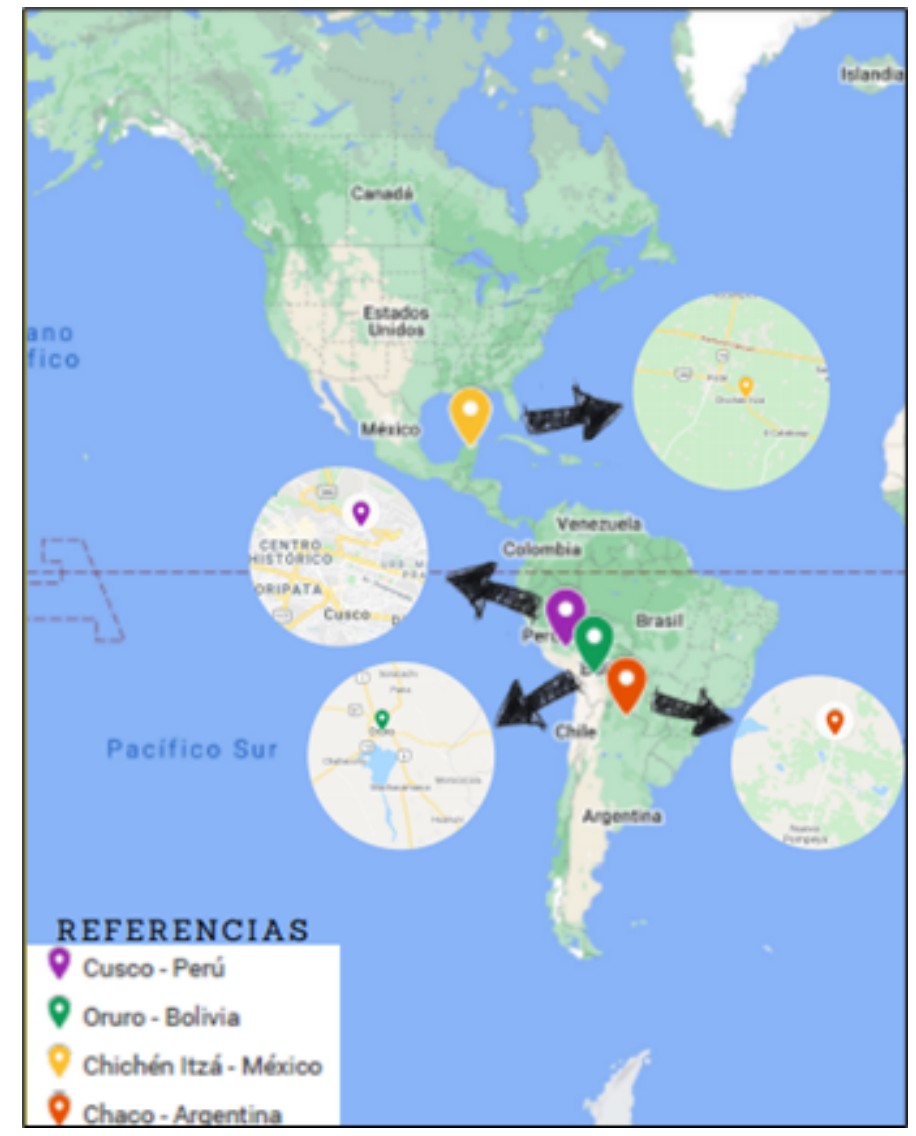


No obstante, consideramos la categoría de drama social por dos motivos: el primero tiene que ver con las distintas temporalidades que se dan en los viajes y los consecuentes encuentros con diversos grupos culturales. Es decir, existe un tiempo en el que se realizó el viaje; un tiempo liminal ante la ruptura de los paisajes observados en los viajes; y un tiempo en el que esas rupturas quedan representadas a partir del diálogo-performance, que se narra desde el presente sobre aquella acción-recuerdo.

El segundo motivo está compuesto de un tiempo pasado, un tiempo futuro pasado y un tiempo presente. Los viajes representaron rupturas por las cuales Marta Tomé se preguntó por la pobreza y las injusticias sociales. Esto llevó a que comenzara a militar con el Movimiento de Sacerdotes del Tercer Mundo (MSTM), para fines de la década de los sesenta. Hablamos de un pasado como aquel tiempo en el que ocurrió la acción; un futuro pasado, en el que se pensó que había que cambiar esas injusticias para las generaciones venideras; y un presente histórico situado desde el que se narra y reflexiona sobre aquellas acciones-recuerdos. Este segundo motivo también tiene un anclaje en cómo se relatan estas experiencias, pues representan una ruptura dentro del proceso social y las contingencias políticas:

M: Cuando entré a la Catedral de Cusco sentí que eso fue meterles la pata encima. No tiene nada que ver con la evangelización. En Perú tuve una crisis importante respecto a lo religioso. No sé si a lo religioso. A la Iglesia, que es otra cosa. Cuando estaba en Cusco, estaba la Catedral de Cusco metida encima de la plataforma de los incas que habían hecho la otra. Entonces fui ahí a un confesionario que estaba allí y le hablé al cura... (Entrevista, martes 16 de abril de 2019. Espacio: Casa de Marta Tomé, Villa Lugano).

El fragmento que se cita tiene que ver con el momento en el que Marta Tomé llega a Cusco, y vive, tal como describe, una crisis respecto de las acciones llevadas a cabo en el tiempo de la colonización española por parte de la Iglesia Católica. En este caso, siguiendo el concepto de drama social propuesto por Turner, en la experiencia que relata Tomé se da una ruptura con la Iglesia que sobreviene a una fase de crisis, que muchas veces se acompaña de un momento liminal, sin llegar a ser tal. Es decir, es un momento umbral entre dos fases del proceso social. El fragmento descripto da cuenta de que, dentro de la fase de crisis, hay un momento umbral que se dio a partir de la ruptura del paisaje, al ver las construcciones de la Iglesia Católica sobre las edificaciones que anteriormente habían realizado los incas. Este momento de suspenso permitió que Marta Tomé pudiera cuestionarse acerca de los problemas sociales en Latinoamérica:

M: Hablando con el cura, que creo que estaba también cuestionado, me dice: "Bueno, por lo menos cuando salga trate bien a la gente”, me dice, ¿no? A los pobres se los veía arrastrar los carros con toda la basura.

Me dije:

"Ya está. Aceptas o no aceptas la pobreza como cosa natural. Yo no la acepté." (Entrevista, martes 16 de abril de 2019.

Espacio: Casa Marta Tomé, Villa Lugano).

Esta segunda parte del fragmento tiene relación con la fase que Turner menciona como la acción de desagravio (Turner, 2002). El hecho de hablar con el cura de la Iglesia, y contarle la ruptura generada ante lo visto, es decir la acción colonizadora observada en el paisaje de una construcción edilicia por encima de otra, llevó a que Marta Tomé comenzara a tomar compromiso sobre las acciones políticas, sociales, culturales y económicas. De acuerdo con Kusch:

El paisaje subvierte así el sentido del ser. Le opone al ser, al espejo cristalino de su mundo ordenado, la sinrazón que lo quiebra por rebeldía y autismo, por una imitatio dei que encierra en su seno los vectores de infinitas posibilidades de existencia (Kusch, 2000, p.26).

Un lugar cargado con temporalidad se destaca por su manera única dentro del paisaje que lo rodea. En este caso, la iglesia se extiende a lo largo de generaciones humanas. Su temporalidad no puede observarse por fuera del entorno en el que habitamos. Tal como lo señala Tomé, la iglesia entierra sus raíces en el suelo. La iglesia también resuena a los ciclos de la subsistencia y la vida humana. De acuerdo con Ingold, entre los habitantes de un vecindario la iglesia no es sólo vista sino también oída, a medida que su campana anuncia las estaciones, los meses, los nacimientos, matrimonios y muertes. En otras palabras, como rasgos de un paisaje, la iglesia 
forma parte de los auténticos monumentos del paso del tiempo (Ingold, 2013). El paisaje no es una totalidad a la que cualquiera pueda mirar; es más bien el mundo en donde nos paramos para tener un punto de vista de nuestros alrededores.

En América profunda, Kusch menciona su análisis de lo americano a partir de sus intuiciones en el paisaje. Los viajes al altiplano y la investigación sobre religión precolombina de las zonas quechua y aymara fueron las rupturas que le permitieron pensar en las categorías de lo americano. Esa intuición a la que Kusch se refiere oscila entre el ser y el estar (Kusch, 1999). Es decir, el ser alguien que está aquí, que se considera como una modalidad profunda de la cultura precolombina. Estos dos conceptos forman parte de las raíces de nuestros procesos políticos identitarios. En Cusco, dice el autor, la ciudad sagrada se encuentra llena de adoratorios, que combinan tiempos y espacios, como corresponde a toda revelación (Kusch,1999).

El paisaje, tomando prestada una frase de Merleau-Ponty, "no es el objeto sino el hogar de nuestros pensamientos" (Merleau-Ponty, 1962,p. 24). Esta manera de interpretar el viaje permite cobrar y recobrar conciencia de qué, quiénes y cómo somos. Por esto, en líneas anteriores hablamos de diversas ontologías, que hacen del entorno una agencia, y no un elemento abstracto sin capacidad (Pels, 1998). Los viajes, son los que llevaron a Marta Tomé a comprometerse con la militancia, resistencia y lucha, que hacen a las experiencias desde abajo. Entre ellas, la educación intercultural. Este desde abajo, da cuenta de que en los territorios estas experiencias no tienen relación con una sola problemática, sino con muchas problemáticas que hacen a los tiempos y espacios.

En este sentido, a partir de la observación de un paisaje se genera una multiplicidad de tiempos, que se entraman para que aquella crisis se convierta en un compromiso de justicia social. De acuerdo con Reyes Tovar y Lamy, es importante considerar el paisaje como una unidad espacial, que enmarca el ámbito sensible de los lugares vividos y evidencia las transformaciones sociales. Los territorios y las identidades están dados por la memoria de los espacios en las relaciones: memoria-narrativa, narrativa-lugar y memoria-lugar, que permiten crear un recorrido hacia la forma en el cual se construye el sentido de pertenencia e identificación de los lugares, más allá de sus fronteras físicas (Reyes Tovar y Lamy, 2017). En otras palabras, dentro de estos relatos se presentan como parte del pasado: la sociedad inca, la colonización española y la evangelización de la población. Las formas de entender el territorio están dadas desde una dimensión espacio-temporal. El territorio se transfigura en diversos tiempos: uno pasado, uno presente, uno del recuerdo y uno vivencial.

Retomando la propuesta de Turner, los momentos de las fases de quiebre y crisis se producen durante el viaje, cuando Marta Tomé observa, en el paisaje, las construcciones edilicias de los distintos grupos sociales, una por encima de otra. La fase de desagravio se da hablando con el cura, y finalmente hay una cuarta fase, que Turner (2002) llama reintegración. El "no aceptar la pobreza" nos lleva a dar cuenta de esta reintegración al proceso social y a las miradas que desde esta ruptura fue tomando a lo largo de su vida respecto de los diversos procesos interculturales e interreligiosos, que la misma Iglesia Católica presenta como institución y que permitió el consecuente acercamiento de Tomé a la militancia en el Movimiento de Sacerdotes para el Tercer Mundo (MSTM).

No obstante, la relación de las fases del proceso social y los hechos de la vida de Tomé representan una manera de ser-estar (Kusch, 1999) en el sistema-mundo, ya que existen interacciones desde las distintas cosmovisiones culturales, que se tornan hacia los lugares que toman significado desde la construcción de las relaciones personales, familiares y grupales. En este sentido, entendemos que, si bien hay paisajes que dejamos de habitar físicamente, esto no lleva a que no podamos habitarlos desde la memoria. En este caso, ello permite crear un viaje específico hacia la relación entre el sujeto y el paisaje, el recuerdo y la nostalgia. El paisaje es aquel espacio en el que se toma conciencia de los procesos y se buscan los interrogantes de las raíces que faltan (Kusch, 2000).

Los no lugares forman parte de estos viajes que pertenecen a la biografía de vida que se narra desde este presente. Volver sobre un lugar o paisaje en el que alguna vez se estuvo implica, desde el presente situado, observarlo e interpretarlo desde otro lugar que resignifica ese lugar en otro tiempo. En este sentido, 
consideramos el lugar antropológico, ya que da cuenta de la construcción concreta y simbólica de los espacios y sus tiempos (Grimson y Semán, 2007).

En tanto referencia de los lugares que visitamos en un tiempo determinado, los paisajes nos llevan a pensar en los lugares como un almacén de la memoria: "la identidad del paisaje se deriva, de las múltiples memorias y significados asociados a determinadas locaciones, que confieren un sentimiento de pertenencia y apego a sus habitantes (Skewes, Guerra, Rojas y Mellado, 2011).

Mediante el lado sensible del paisaje podemos establecer narrativas del cómo se viven y se sienten los movimientos en los espacios, lo que permite ir más allá de su visión geométrica y volcar la mirada hacia las palabras, emociones, afectos, ensoñaciones y recuerdos de lo que significa para los sujetos, los imaginarios geográficos del paisaje, los cuales, con base en Debarbieux (1995), fundamentan la experiencia sensible y emocional del razonamiento geográfico.

Las personas "viven" una relación con su entorno. Estos son hechos culturales y se relacionan con determinadas características. Existe un yo que narra y otros que reciben esa narrativa. Desde una perspectiva transversal, consideramos que la narrativa de vida se entrama con la narrativa de las biografías de la cultura material, que puede abordarse por medio de objetos, lugares o paisajes.

En este caso, la biografía de vida de esta educadora se construye a través de la cultura material. Las acciones deliberadas de los seres humanos sobre el mundo material ponen en funcionamiento realidades mentales, representaciones, juicios y principios de pensamiento que llevan a determinadas intuiciones, que hacen a otras formas de conocer y de construir conocimiento. Las narrativas no se silencian y existe una temporalidad en las narrativas de los materiales, que es dinámica, heterogénea y continua.

La articulación entre el aquí y el allá mediante el uso de la imaginación creativa y la experiencia territorial (Lindón, 2008; Cortes, 2009; Hendel, 2020) nos lleva a pensar en el paisaje como un espacio creativo, que remite a la construcción simbólica tanto material como inmaterial por parte de sus actores. Permite destacar cómo, con un proceso de movilidad, los sujetos llevan consigo un conjunto de símbolos de información sobre el lugar del cual parten y posteriormente, en su lugar de origen, pueden crear traducciones, acomodos, mezclas u olvidos de aquellos; así como al regresar a su lugar cotidiano, establecen un proceso de intercambio intercultural, y en este caso, interreligioso. Las configuraciones emotivas y físicas del paisaje forman parte del cúmulo de percepciones y sentidos que se hacen visibles en el paisaje, lo que causa un contraste de miradas que derivan en una diversidad de significados.

Según Debarbieux, la temporalidad se encuentra presente en la conformación de las prácticas y representaciones espaciales de una sociedad hacia la búsqueda de significaciones. Para Ernest Bloch, el viaje auténtico tiene como efecto no sólo una modificación del lugar sino también del viajero mismo, que permite poner de relieve la experiencia para proporcionar un conocimiento del mundo, que existe de manera fragmentaria y que guarda los silenciamientos sobre los distintos procesos que transcurren. Por ello, no podemos hablar de una razón unidisciplinaria sobre aquellos conocimientos que se construyen ajenos a los sujetos (Bloch, 1973). En este sentido, recuperamos el recuerdo de cuando Marta Tomé llega a Bolivia junto con una compañera de la Acción Católica (ACA):

M: Bueno, entonces te tenías que desacostumbrar a todo, viste, y volver a armar. Entonces, cuando llegamos a Oruro era el Carnaval. Porque la hermana de la que era compañera mía de viaje era profesora de geografía. Yo no tenía ni idea de que existía el carnaval de Oruro, me entendés, en ese momento. Fue ella la que diseñó el viaje.

Entonces, nosotras llegamos allá justamente la noche anterior. El presidente de Bolivia también llegó allá. Te aclaro que en ese hotel no había agua. Pero era el hotel donde estaba el presidente también, viste. Bueno, entonces estábamos esperand o la cena y creo que iban a traer un pollo. Entonces mi compañera me dijo:

- "Mirá, si cuando venga el pollo vos me vas a hablar de la pobreza, te voy a poner el plato arriba en la cabeza".

O sea que yo ya estaba hablando permanentemente de la pobreza. Sí, no podía aceptar tantas diferencias (Entrevista, martes 16 de abril de 2019. Espacio: Casa de Marta Tomé, Villa Lugano). 
Este fragmento da cuenta del reordenamiento de la alteridad, lo cual no va a significar ajenidad, debido a que los mundos simbólicos nos construyen (González Rodríguez y Smith, 1998). Estos redescubrimientos invitan a pensar en la interculturalidad. Si bien Marta Tomé ya se había cuestionado acerca de la pobreza, esta idea es constante a lo largo de su trayectoria de vida, y la llevó a pensar y generar proyectos que trabajaran sobre las problemáticas como la desigualdad, la injusticia social; y a luchar por la igualdad de condiciones y los derechos sociales y humanos.

Los viajes implican una metáfora relacional, una instancia de mediación para producir acercamientos basados en el entendimiento, y en los nuevos procesos de neocolonización y de desencuentro contemporáneo. El viaje es un recorrido simbólico que permite un desanclaje para recrear la mirada del conocimiento: "La construcción de lo mediacional como forma de crear proximidades” (González Rodríguez y Smith, 1998, p. 481).

$\mathrm{Al}$ año siguiente, en el viaje por México (1968), Tomé sigue preguntándose por la pobreza, pero aquí ya reconoce que las identidades y memorias de los grupos sociales son un eje presente en los territorios que visita:

M: Bueno, entonces, lo mismo hice al año siguiente: las tres juntas, ya con la profesora de geografía, hicimos el viaje a México. Ahí lo que me llamó la atención, además de la pobreza, es que ellos parecían orgullosos de su pasado, cosa que no parecía que se diera en Bolivia y Perú (Entrevista, martes 16 de abril de 2019. Espacio: Casa de Marta Tomé, Villa Lugano).

De acuerdo con Krotz, en los viajes el asombro juega un papel relevante, a tal grado que es el elemento que caracteriza cualquier viaje digno de tal término, no sólo en su fase inicial sino también a lo largo de todo su transcurso y hasta la reflexión posterior (Krotz, 1991). Este asombro sería, según Bloch, un lugar de la anticipación, donde se abarca la realidad, que es totalizador, teórico y práctico a la vez (Bloch, 1973; citado en Krotz et al., 1991), que comprende el pensar y sentir, ver y ansiar; y que constituye las diversas maneras de conocer y entender los mundos.

\section{A MODO DE CONCLUSIÓN}

La biografía de vida de la educadora Marta Tomé se encuentra atravesada por distintas rupturas que hacen a la construcción de las memorias sociales y territorializadas, y en este caso, también forman parte de los antecedentes de la historización de la educación intercultural en la Argentina. Los viajes por Latinoamérica representan rupturas transformativas clave en lo que luego será la construcción del método de bialfabetización y la pareja pedagógica.

Asimismo, en este caso, buscamos encontrar a partir de los viajes distintas aristas que se pueden observar a través de la cultura material, es decir, a partir de los objetos, lugares y paisajes. Su paso por Cusco es determinante a la hora de pensar en la biografía social de los objetos, espacios y paisajes. Esto nos lleva a considerar que el trabajo con las biografías de vida no sólo implica el narrar los hitos de vida que hacen a la persona, sino también dar cuenta de cómo aquellos acontecimientos que forman parte de su vida se entraman con una cultura material, que hace a otras formas de conocer. En este caso, forman parte de las rupturas transformativas que llevan a pensar en lo que fueron experiencias territorializadas que son antecedentes relevantes para la historización de la educación intercultural en la Argentina.

\section{REFERENCIAS}

Appadurai, A. (1996). Introducción: Las mercancías y la política de valor. En La vida social de las cosas, pp. 17-89. Londres: Cambridge University Press. (Original publicado en 1991).

Bertaux, D. (2005). Los relatos de vida. Perspectiva etnosociológica. Madrid: Edicions Bellaterra.

Bloch, E. (1973). Geist der Utopie. Francfort: Suhrkamp 
Bourdieu, P. (1989). La ilusión biográfica. Historia y Fuente Oral, 2. Universidad de Barcelona. DOI: http://dx.doi. org/10.22201/fcpys.24484938e.2011.56.29460

Clifford, J. (1999). Prácticas espaciales: el trabajo de campo, el viaje y la disciplina de la antropología. En Itinerarios transculturales (pp.71-120). Barcelona: Gedisa.

Cortes, G. (2009). Migraciones, construcciones transnacionales y prácticas de circulación. Un enfoque desde el territorio. Párrafos Geográficos, 8(1), 35-53.

Debarbieux, B. (1995). Le lieu, le territoire et trois figures de rhétorique. L'espace géographique, 24(2), 97-112.

Escobar, A. (2014). Sentipensar con la tierra. Nuevas lecturas sobre desarrollo, territorio y diferencia. Medellín: Ediciones UNAULA.

Gómez, H. (2000). De los lugares y sentidos de la memoria. En C. Gnecco y M. Zambrano, Memorias hegemónicas, memorias disidentes, el pasado como politica (pp.23-53). Bogotá: Ministerio de Cultura, Universidad del CaucaInstituto Colombiano de Antropología e Historia.

González Rodríguez, S. y Smith, M. (1998, 9 -13 de noviembre). El viaje: Una metáfora de la Alteridad. Ponencia presentada en III Congreso Chileno de Antropología. Desafíos para el Tercer Milenio, Temuco, Chile.

Grimson, A. y Semán, P. (2007). Los no-lugares: una criatura etnocéntrica. Konvergencias. Filosofía y Culturas en Diálogo, IV(15), 74-76.

Hendel, V. (2020). Cartografías del peligro. Desplazamientos, migración, fronteras y violencias desde la experiencia de los jóvenes en un barrio del Gran Buenos Aires, Argentina (2018-2019). Historia y Sociedad, 39, 184-212. h ttp://dx.doi.org/10.15446/hys.n39.82576

Ingold, T. (2013). La temporalidad del paisaje, pp.1-27. Original publicado en 1993. Trad. Matías Lepori.

Krotz, E. (1991). Viaje, trabajo de campo y conocimiento antropológico. Revista Alteridades, 1(1), 50-57.

Kusch, R. (1999). América profunda. Buenos Aires: Editorial Biblos.

Kusch, R. (2000). Dialéctica del continente mestizo. En Obras Completas, tomo 1 (pp.25-37). Rosario: Fundación Ross.

Lindón, A. (2008). De espacialidades y transnacionalismo. En D. Hiernaux yM. Zárate (Eds.). Espacios y transnacionalismo (pp.119-157). México: Universidad Autónoma Metropolitana Iztapalapa.

Marcus, G.E. \& Fischer, M.M.J. (1986). Anthropology as Cultural Critique: An Experimental Moment in the Human Sciences. Chicago: University of Chicago Press.

Merleau-Ponty, M. (1962). The Phenomenology of Perception. Londres: Routledge \& Kegan Paul. Trad. C. Smith.

Myerhoff, B. (2007). Stories as Equipment for Living: Last Talks and Tales of Barbara Myerhoff. Michigan: University of Michigan Press.

Pels, P. (1998). The spirit of matter. On fetish, rarity, fact, and fancy. En P. Spyer (ed.), Border fetishisms. Material objects in unstable spaces (pp. 91-121). Londres: Routledge.

Piña, C. (1989). Aproximaciones metodológicas al relato autobiográfico. Revista Opciones, 16, mayo-agosto, 107-124.

Reyes Tovar, M. y Lamy, B. (2017). Migración y transformación sociocultural: el paisaje como referente de la movilidad. Acta Universitaria [en línea], 27(3), 91-100. Recuperado de https://www.redalyc.org/articulo.oa?i $\mathrm{d}=41652062009$.

Skewes, J., Guerra, D., Rojas, P. y Mellado, M. A. (2011). ¿La memoria de los paisajes o los paisajes de la memoria? Los enigmas de la sustentabilidad socioambiental en las geografías en disputa. Desenvolvimento e Medio Ambiente, 23, 39-57.

Turner, V. (2002). Dramas sociales y metáforas rituales. En I. Geist (Comp.), Antropología del ritual: Victor Tumer (pp.35-71). México D.F.: INAH-ENAH. (Original publicado en 1974). 